\title{
BibLIOGRAPHY
}

1. L. Fuchs, On subdirect unions. I, Acta Math. Acad. Sci. Hungar. vol. 3 (1952) pp. 103-120.

2. R. Remak, Über dic Darstellung der endlichen Gruppen als Untergruppen direkter Produkte, J. Reine Angew. Math. vol. 163 (1930) pp. 1-44.

AUburn University AND

Louisiana Polytechnic Institute

\section{FREE-ALGEBRAIC CHARACTERIZATIONS OF PRIMAL AND INDEPENDENT ALGEBRAS}

F. M. SIOSON

In the study of many-valued logics, one is led to consider a (finitary) algebra $\left(A, o_{1}, \cdots, o_{n}\right)$, or simply $A$, with a finite number of primitive operations that generate by composition all functions in $A^{A^{m}}$ for each $m<\infty$. Such algebras are called primal. For example, the algebra $(\{0,1\}, \wedge, \sim)$ of truth-values in 2-valued logic and, more generally, $(\{0, \cdots, n-1\}, \min \{x, y\}, x+1(\bmod n))$ in $n$ valued Post logics are primal algebras. The truth-values of the Łukasiewicz-Tarski logics $(\{0, \cdots, n-1\}, C, N)$, where $C x y$ $=\max \{0, y-x\}, N x=n-1-x$, do not form a primal algebra, but if the 0 -ary (constant) operation 1 is admitted, then $(\{0, \cdots, n-1\}$, $C, N, 1)$ becomes a primal algebra. Note that any primal algebra is finite, for if it were infinite, then the set of functions would be uncountable, while the set of generated operations would be countable at most.

If $O_{1}, \cdots, O_{n}$ are the operations symbols in the language for the operations $o_{1}, \cdots, o_{n}$ of a fixed species (or similarity type), then by the absolutely-free algebra (of the given species), $\left(\Phi_{k}, o_{1}, \cdots, o_{n}\right)$, with $k$ generators $x_{1}, \cdots, x_{k}$, is meant the set of all formal expressions defined inductively as follows:

$1^{\circ} . x_{1}, \cdots, x_{k} \in \Phi_{k}$

$2^{\circ}$. for each $i=1, \cdots, n$, if $\phi_{1}, \cdots, \phi_{k_{i}} \in \Phi_{k}$, then also $O_{i}\left(\phi_{1}, \cdots, \phi_{k_{i}}\right) \in \Phi_{k}$;

with the operations defined by setting

$$
o_{i}\left(\phi_{1}, \cdots, \phi_{k_{i}}\right)=O_{i}\left(\phi_{1}, \cdots, \phi_{k_{i}}\right) \text {. }
$$

Received by the editors April 7, 1960 and, in revised form, May 4 and May 2.3, 1960. 
Let $E q_{k}(A)=\left\{\left(\phi, \phi^{\prime}\right) \in \Phi_{k} \times \Phi_{k}\right.$ : for each $\left.h \in \operatorname{Hom}\left(\Phi_{k}, A\right), h(\phi)=h\left(\phi^{\prime}\right)\right\}$. From the fact that a homomorphism determines a congruence (relation) and an intersection of congruences is also a congruence, it follows that $E q_{k}(A)$ is a congruence on $\Phi_{k}$. The free A-algebra with $k$ generators $x_{1}, \cdots, x_{k}$ is then defined as the quotient algebra $F_{k}(A)$ $=\Phi_{k} / E q_{k}(A)$. Following Foster [4] we write $\phi \equiv \phi^{\prime}(A)$ if $\left(\phi, \phi^{\prime}\right)$ $\in E q_{k}(A)$ for some $k$. It is easy to see that $F_{k}(A)$ is essentially the algebra of all $k$-ary (compositional) operations in $A$.

Several characterizations of primal algebras are known $[1 ; 4 ; 6]$. We give another one here.

TheOREM 1. An algebra $\left(A, o_{1}, \cdots, o_{n}\right)$ is primal iff

$$
F_{2}(A) \cong A^{a^{2}},
$$

where the cardinal of $A$ is $|A|=a<\infty$ and $A^{a^{2}}$ is the direct product of $a^{2}$ copies of $A$.

Proof. Foster [3] proved the necessity part of this theorem by using his fundamental structure theorem for primal algebras. A proof independent of this result is easily reproduced.

From perfectly general considerations (see Foreword [2]), it is well known that $F_{2}(A)$ is isomorphic with a subalgebra of the direct power $A^{a^{2}}$, where $|A|=a$, and hence $\left|F_{2}(A)\right| \leqq a^{a^{2}}$. Now suppose further $A$ is primal and hence finite; then each binary function $f \in A^{\Lambda^{2}}$ is represented by an equivalence class of elements $\phi$ from $\Phi_{2}$. Whence $a^{a^{2}}=\left|A^{A^{2}}\right| \leqq\left|F_{2}(A)\right|$ and therefore $F_{2}(A) \cong A^{a^{2}}$.

Conversely, suppose $F_{2}(A) \cong A^{a^{2}}$, where $|A|=a<\infty$. Each equivalence class $E(\phi)$ in $F_{2}(A)$ defines a function $f$ of two variables on $A$. By virtue of the given isomorphism, the correspondence $E(\phi) \rightarrow f$ is onto and hence each binary function $f$ is represented by a composition of the fundamental operations. Binary functions on any set generate by composition all finitary functions (see [5]); whence primality follows.

COROLlaRy 1. An algebra $\left(A, o_{1}, \cdots, o_{n}\right)$ is primal iff

$$
F_{k}(A) \cong A^{a k},
$$

where $k \geqq 2$ and $|A|=a<\infty$.

Another notion quite strongly tied up with the notion of primality is that of independence. A class of algebras of the same species is independent iff for every finite number of them $A_{1}, \cdots, A_{m}$ and every set of expressions $\phi_{1}, \cdots, \phi_{m}$ there exist another expression $\psi$ such that $\phi_{1} \equiv \psi\left(A_{1}\right), \cdots, \phi_{m} \equiv \psi\left(A_{m}\right)$. 
We also give a characterization of this concept in terms of free algebras.

Theorem 2. $A$ finite set $A_{1}, \cdots, A_{m}$ of finite algebras of the same species is independent iff

$$
F_{m}\left(A_{1} \times \cdots \times A_{m}\right) \cong F_{m}\left(A_{1}\right) \times \cdots \times F_{m}\left(A_{m}\right) .
$$

Proof. The following lemma will be used in proving the necessity and sufficiency parts of the theorem and also Theorem 3.

Lemma 1. The set $S=\left\{\left(E_{1}(\phi), \cdots, E_{m}(\phi)\right): \phi \in \Phi_{m}\right\}$, where $E_{i}(\phi)$ denotes the equivalence class in $F_{m}\left(A_{i}\right)$ containing $\phi$, forms a subalgebra of $F_{m}\left(A_{1}\right) \times \cdots \times F_{m}\left(A_{m}\right)$ isomorphic with $F_{m}\left(A_{1} \times \cdots \times A_{m}\right)$.

Proof of the Lemma. $S$ is clearly a subset of $F_{m}\left(A_{1}\right) \times \cdots$ $\times F_{m}\left(A_{m}\right)$; and for any operation $o_{i}$, which is $k_{i}$-ary,

$$
\begin{aligned}
o_{i}\left[\left(E_{1}\left(\phi_{1}\right),\right.\right. & \left.\left.\cdots, E_{m}\left(\phi_{1}\right)\right), \cdots,\left(E_{1}\left(\phi_{k_{i}}\right), \cdots, E_{m}\left(\phi_{k_{i}}\right)\right)\right] \\
& =\left(o_{i}\left(E_{1}\left(\phi_{1}\right), \cdots, E_{1}\left(\phi_{k_{i}}\right)\right), \cdots, o_{i}\left(E_{m}\left(\phi_{1}\right), \cdots, E_{m}\left(\phi_{k_{i}}\right)\right)\right) \\
& =\left(E_{1}\left(o_{i}\left(\phi_{1}, \cdots, \phi_{k_{i}}\right)\right), \cdots, E_{m}\left(o_{i}\left(\phi_{1}, \cdots, \phi_{k_{i}}\right)\right)\right) \\
& =\left(E_{1}(\phi), \cdots, E_{m}(\phi)\right) \in S .
\end{aligned}
$$

Hence $S$ is a subalgebra of $F_{m}\left(A_{1}\right) \times \cdots \times F_{m}\left(A_{m}\right)$.

Consider now the mapping $f: F_{m}\left(A_{1} \times \cdots \times A_{m}\right) \rightarrow S$ defined by $f(E(\phi))=\left(E_{1}(\phi), \cdots, E_{m}(\phi)\right)$. This is onto $S$ and also 1-1, since if $f(E(\phi))=f\left(E\left(\phi^{\prime}\right)\right)$, then $E_{i}(\phi)=E_{i}\left(\phi^{\prime}\right)$ or $\phi \equiv \phi^{\prime}\left(A_{i}\right)$ for each $i=1, \cdots, m$ and hence $\phi \equiv \phi^{\prime}\left(A_{1} \times \cdots \times A_{m}\right)$ or $E(\phi)=E\left(\phi^{\prime}\right)$. In addition,

$$
\begin{aligned}
f\left[o_{i}\left(E\left(\phi_{1}\right), \cdots, E\left(\phi_{k_{i}}\right)\right)\right]=f\left[E\left(o_{i}\left(\phi_{1}, \cdots, \phi_{k_{i}}\right)\right)\right] \\
\quad=\left(E_{1}\left(o_{i}\left(\phi_{1}, \cdots, \phi_{k_{i}}\right)\right), \cdots, E_{m}\left(o_{i}\left(\phi_{1}, \cdots, \phi_{k_{i}}\right)\right)\right) \\
\quad=\left(o_{i}\left(E_{1}\left(\phi_{1}\right), \cdots, E_{1}\left(\phi_{k_{i}}\right)\right), \cdots, o_{i}\left(E_{m}\left(\phi_{1}\right), \cdots, E_{m}\left(\phi_{k_{i}}\right)\right)\right) \\
\quad=o_{i}\left(f\left(E\left(\phi_{1}\right)\right), \cdots, f\left(E\left(\phi_{k_{i}}\right)\right)\right) .
\end{aligned}
$$

Thus we have $F_{m}\left(A_{1} \times \cdots \times A_{m}\right) \cong S$.

To continue with the proof of the theorem, let now

$$
\left(E_{1}\left(\phi_{1}\right), \cdots, E_{m}\left(\phi_{m}\right)\right) \in F_{m}\left(A_{1}\right) \times \cdots \times F_{m}\left(A_{m}\right) .
$$

If $A_{1}, \cdots, A_{m}$ are independent, then corresponding to the expressions $\phi_{1}, \cdots, \phi_{m}$ there exists another $\psi$ such that $\phi_{1} \equiv \psi\left(A_{1}\right), \cdots, \phi_{m}$ $\equiv \psi\left(A_{m}\right)$. Hence $\left(E_{1}\left(\phi_{1}\right), \cdots, E_{m}\left(\phi_{m}\right)\right)=\left(E_{1}(\psi), \cdots, E_{m}(\psi)\right)$. This implies $F_{m}\left(A_{1}\right) \times \cdots \times F_{m}\left(A_{m}\right) \subseteq S$. The other inclusion is obvious by Lemma 1 ; whence $S=F_{m}\left(A_{1}\right) \times \cdots \times F_{m}\left(A_{m}\right)$, from which the 
result is now clear by Lemma 1.

Conversely, suppose $A_{1}, \cdots, A_{m}$ are finite algebras such that $F_{m}\left(A_{1} \times \cdots \times A_{m}\right) \cong F_{m}\left(A_{1}\right) \times \cdots \times F_{m}\left(A_{m}\right)$. By Lemma 1 , $S \cong F_{m}\left(A_{1} \times \cdots \times A_{m}\right)$. All algebras are finite and hence $S=F_{m}\left(A_{1}\right) \times \cdots \times F_{m}\left(A_{m}\right)$. Thus, if there are given $\phi_{1}, \cdots, \phi_{m}$ $\in \Phi_{m}$, then $\left(E_{1}\left(\phi_{1}\right), \cdots, E_{m}\left(\phi_{m}\right)\right)$ is in $S$, whence there exists an expression $\psi$ such that $\left(E_{1}(\psi), \cdots, E_{m}(\psi)\right)=\left(E_{1}\left(\phi_{1}\right), \cdots, E_{m}\left(\phi_{m}\right)\right)$, or in other words, $\phi_{i} \equiv \psi\left(A_{i}\right)$ for each $i=1, \cdots, m$. Now choose $\phi_{i}$ $=x_{i}, i=1, \cdots, m$; then for every set of arbitrary expressions $\phi_{1}^{\prime}, \cdots, \phi_{m}^{\prime}$, we get $\psi\left(\phi_{1}^{\prime}, \cdots, \phi_{m}^{\prime}\right) \equiv \phi_{i}^{\prime}\left(A_{i}\right), i=1, \cdots, m$.

Corollary 2. A family of finite algebras of the same species is independent iff for every finite subfamily $A_{1}, \cdots, A_{m}$,

$$
F_{m}\left(A_{1} \times \cdots \times A_{m}\right) \cong F_{m}\left(A_{1}\right) \times \cdots \times F_{m}\left(A_{m}\right) .
$$

Finally, we give a characterization of primal clusters, i.e., independent classes of primal algebras. For their significance see Foster's works.

Theorem 3. A family of pairwise nonisomorphic algebras of the same species forms a primal cluster iff for every finite subfamily $A_{1}, A_{2}$, $\cdots, A_{m}$ with $\left|A_{i}\right|=a_{i}, i=1, \cdots, m$,

$$
F_{m}\left(A_{1} \times \cdots \times A_{m}\right) \cong A_{1}^{a_{1}^{m}} \times \cdots \times A_{m}^{a_{m}^{m}} .
$$

Proof. If $A_{1}, \cdots, A_{m}$ is any finite subfamily of the given family, then by independence, it follows by Theorem 2 that

$$
F_{m}\left(A_{1} \times \cdots \times A_{m}\right) \cong F_{m}\left(A_{1}\right) \times \cdots \times F_{m}\left(A_{m}\right) .
$$

By primality of the algebras and Theorem 1 , then $F_{m}\left(A_{1} \times \cdots \times A_{m}\right)$ $\cong A_{1}^{a_{n}^{m}} \times \cdots \times A_{m}^{a_{m}^{m}}$.

Conversely, we know from Lemma 1 that $F_{m}\left(A_{1} \times \cdots \times A_{m}\right)$ is isomorphic with a subalgebra $S$ of $F_{m}\left(A_{1}\right) \times \cdots \times F_{m}\left(A_{m}\right)$. Each tuple $\left(E_{1}\left(\phi_{1}\right), \cdots, E_{m}\left(\phi_{m}\right)\right) \in F_{m}\left(A_{1}\right) \times \cdots \times F_{m}\left(A_{m}\right)$ defines an $m-$ tuple of functions $\left(f_{1}, \cdots, f_{m}\right) \in A_{1}^{A_{1}^{m}} \times \cdots \times A_{m^{m}}^{A_{m}^{m}}$. Thus by virtue of the given isomorphism and the finiteness of the algebras, the correspondence $\left(E_{1}\left(\phi_{1}\right), \cdots, E_{m}\left(\phi_{m}\right)\right) \rightarrow\left(f_{1}, \cdots, f_{m}\right)$ is 1-1 and onto. By the same argument featured in the proof of Theorem 1, it follows that $A_{i}$ is a primal algebra for each $i=1, \cdots, m$. By Corollary 1 , $F_{m}\left(A_{i}\right) \cong A_{i}^{a^{m}}$ for each $i=1, \cdots, m$ and therefore we finally arrive at $F_{m}\left(A_{1} \times \cdots \times A_{m}\right)$

$$
\cong A_{1}^{a_{1}^{m}} \times \cdots \times A_{m}^{a_{m}{ }^{2}} \cong F_{m}\left(A_{1}\right) \times \cdots \times F_{m}\left(A_{m}\right) .
$$


Independence now follows by virtue of Theorem 2 and hence the given family is a primal cluster.

\section{REFERENCES}

1. S. V. Ablonskij, Functional constructions in many-valued logics, Trudy Tret'ego Vsesoyuznogo Matematičeskogo s"ezda, Moskva, 1956, Moscow, Izdat. Akad. Nauk SSSR, vol. 2, 1956, pp. 71-73.

2. G. Birkhoff, "Foreword," Lattice theory, New York, Amer. Math. Soc. Colloquium Publications, vol. 25, 1948.

3. A. L. Foster, On the finiteness of free universal algebras, Proc. Amer. Math. Soc. vol. 7 (1956) pp. 1011-1013.

4. - The generalized Chinese remainder theorem for universal algebras, Math. Z. vol. 56 (1957) pp. 452-469.

5. W. Sierpinski, Sur les fonctions de plusieurs variables, Fund. Math. vol. 33 (1945) pp. 169-173.

6. Jerzy Słupecki, Kryterium petnosci wielowartosciowych systemow logiki zdan ( $A$ criterion of the functional completeness of many-valued logics), Comptes Rendus de la Société des Sciences et des Lettres de Varsovie, III, vol. 32 (1939) pp. 102-110.

UNIVERSITY OF CALIFORNia 